

\section{Linear Operators and Their Essential Pseudospectra}


$\Longrightarrow$ Taylor \& Francis

Taylor \& Francis Group

http://taylorandfrancis.com 


\section{Linear Operators and Their Essential Pseudospectra}

Aref Jeribi, PhD

Department of Mathematics,

University of Sfax, Tunisia

E-mail:aref.jeribi@fss.rnu.tn

$10 \begin{aligned} & \text { APPLE } \\ & \text { ACADEMIC } \\ & \text { PRESS }\end{aligned}$ 
Apple Academic Press Inc. 3333 Mistwell Crescent Oakville, ON L6L 0A2 Canada
Apple Academic Press Inc.

9 Spinnaker Way

Waretown, NJ 08758 USA

(C) 2018 by Apple Academic Press, Inc.

Exclusive worldwide distribution by CRC Press, a member of Taylor \& Francis Group

No claim to original U.S. Government works

Printed in the United States of America on acid-free paper

International Standard Book Number-13: 978-1-77188-699-4 (Hardcover)

International Standard Book Number-13: 978-1-351-04627-5 (eBook)

All rights reserved. No part of this work may be reprinted or reproduced or utilized in any form or by any electric, mechanical or other means, now known or hereafter invented, including photocopying and recording, or in any information storage or retrieval system, without permission in writing from the publisher or its distributor, except in the case of brief excerpts or quotations for use in reviews or critical articles.

This book contains information obtained from authentic and highly regarded sources. Reprinted material is quoted with permission and sources are indicated. Copyright for individual articles remains with the authors as indicated. A wide variety of references are listed. Reasonable efforts have been made to publish reliable data and information, but the authors, editors, and the publisher cannot assume responsibility for the validity of all materials or the consequences of their use. The authors, editors, and the publisher have attempted to trace the copyright holders of all material reproduced in this publication and apologize to copyright holders if permission to publish in this form has not been obtained. If any copyright material has not been acknowledged, please write and let us know so we may rectify in any future reprint.

Trademark Notice: Registered trademark of products or corporate names are used only for explanation and identification without intent to infringe.

\section{Library and Archives Canada Cataloguing in Publication}

\footnotetext{
Jeribi, Aref, author

Linear operators and their essential pseudospectra / Aref Jeribi, PhD (Department of Mathematics, University of Sfax, Tunisia).
}

Includes bibliographical references and index.

Issued in print and electronic formats.

ISBN 978-1-77188-699-4 (hardcover).--ISBN 978-1-351-04627-5 (PDF)

1. Linear operators. 2. Spectral theory (Mathematics). I. Title.

$$
\text { QA329.2.J47 } 2018 \quad \text { 515'.7246 C2018-900406-1 C2018-900407-X }
$$

CIP data on file with US Library of Congress

Apple Academic Press also publishes its books in a variety of electronic formats. Some content that appears in print may not be available in electronic format. For information about Apple Academic Press products, visit our website at www.appleacademicpress.com and the CRC Press website at www.crcpress.com 
my mother Sania, my father Ali, my wife Fadoua, my children Adam and Rahma, my brothers Sofien and Mohamed Amin, my sister Elhem, and all members of my extended family 
$\Longrightarrow$ Taylor \& Francis

Taylor \& Francis Group

http://taylorandfrancis.com 


\section{Contents}

About the Author xiii

Preface $\quad$ Xv

1 Introduction 1

1.1 Essential Spectra and Relative Essential Spectra . . . . 2

1.2 Essential Pseudospectra . . . . . . . . . . . . 8

1.3 Structured Essential Pseudospectra and Relative Structured Essential Pseudospectra . . . . . . . . . . . 12

1.4 Condition Pseudospectrum . . . . . . . . . . . . . . . . . . . . . . . . 14

1.5 Outline of Contents . . . . . . . . . . . . 15

2 Fundamentals 19

2.1 Operators . . . . . . . . . . . . . . . . 19

2.1 .1 Linear Operators . . . . . . . . . . . . . . . . . . . . . . . . . . . .

2.1.2 Bounded Operators . . . . . . . . . . . . . 20

2.1.3 Closed and Closable Operators . . . . . . . . . 20

2.1 .4 Adjoint Operator . . . . . . . . . . . . . . . . . . 22

2.1 .5 Direct Sum . . . . . . . . . . . . . . . 24

2.1.6 Resolvent Set and Spectrum . . . . . . . . . . . . 25

2.1 .7 Compact Operators . . . . . . . . . . . . 26

2.1.8 A-Defined, $A$-Bounded, and A-Compact Operators 27

2.1.9 Weakly Compact and $A$-Weakly Compact Operators . . . . . . . . . . . . . . . . . 29

2.1 .10 Dunford-Pettis Property . . . . . . . . . . . . 29

2.1 .11 Strictly Singular Operators . . . . . . . . . . 30 
2.1.12 Strictly Cosingular . . . . . . . . . . . . . 31

2.1 .13 Perturbation Function . . . . . . . . . . . . . . 32

2.1.14 Measure of Non-Strict-Singularity . . . . . . . . 33

2.1 .15 Semigroup Theory . . . . . . . . . . . . . 33

2.2 Fredholm and Semi-Fredholm Operators _ . . . . . . . 34

2.2.1 Definitions . . . . . . . . . . . . . . 34

2.2.2 Basics on Bounded Fredholm Operators . . . . . . 37

2.2.3 Basics on Unbounded Fredholm Operators . . . . 43

2.3 Perturbation . . . . . . . . . . . . . . . . . . . . . 48

2.3.1 Fredholm and Semi-Fredholm Perturbations . . . . 48

2.3.2 Semi-Fredholm Perturbations . . . . . . . . . 51

2.3.3 Riesz Operator . . . . . . . . . . . . . . . . . . . . . . 52

2.3.4 Some Perturbation Results . . . . . . . . . . . . . 53

2.3.5 A-Fredholm Perturbation . . . . . . . . . . . . . 54

2.3.6 A-Compact Perturbations . . . . . . . . . . . . . 55

2.3.7 The Convergence Compactly . . . . . . . . . . . 56

2.4 Ascent and Descent Operators . . . . . . . . . . . . 57

2.4.1 Bounded Operators . . . . . . . . . . . . . . . 57

2.4 .2 Unbounded Operators . . . . . . . . . . . . 62

2.5 Semi-Browder and Browder Operators _ . . . . . . . 63

2.5.1 Semi-Browder Operators . . . . . . . . . . . . 63

2.5.2 Fredholm Operator with Finite Ascent and Descent 65

2.6 Measure of Noncompactness . . . . . . . . . . . . . 67

2.6.1 Measure of Noncompactness of a Bounded Subset 68

2.6.2 Measure of Noncompactness of an Operator . . . . 69

2.6.3 Measure of Non-Strict-Singularity . . . . . . . . 71

2.6.4 $\gamma$-Relatively Bounded . . . . . . . . . . . . 73

2.6.5 Perturbation Result . . . . . . . . . . . . . . . 73

$2.7 \gamma$-Diagonally Dominant . . . . . . . . . 75

2.8 Gap Topology . . . . . . . . . . . . . . . . . . . . . . 76

2.8.1 Gap Between Two Subsets . . . . . . . . . . . . 76

2.8.2 Gap Between Two Operators . . . . . . . . . . . 77 
2.8.3 Convergence in the Generalized Sense . . . . . . 78

2.9 Quasi-Inverse Operator . . . . . . . . . . . . . . . . . . . 79

2.10 Limit Inferior and Superior . . . . . . . . . . . . 83

3 Spectra $\quad 85$

3.1 Essential Spectra . . . . . . . . . . . . . . . 85

3.1.1 Definitions . . . . . . . . . . . 85

3.1.2 Characterization of Essential Spectra . . . . . . 88

3.2 The Left and Right Jeribi Essential Spectra _. . . . . . . 91

$3.3 S$-Resolvent Set, $S$-Spectra, and $S$-Essential Spectra $\ldots .92$

3.3.1 The $S$-Resolvent Set . . . . . . . . . . . . . . 92

3.3.2 S-Spectra . . . . . . . . . . . . . . 98

3.3.3 S-Browder's Resolvent . . . . . . . . . . . . . 100

3.3.4 S-Essential Spectra . . . . . . . . . . . . . 104

3.4 Invariance of the $S$-Essential Spectrum $\ldots \ldots$. . . . 108

3.5 Pseudospectra . . . . . . . . . . . . 113

3.5.1 Pseudospectrum . . . . . . . . . . . . 113

3.5.2 S-Pseudospectrum . . . . . . . . . . . 115

3.5.3 Ammar-Jeribi Essential Pseudospectrum . . . . . . 119

3.5.4 Essential Pseudospectra . . . . . . . . . . . 122

3.5.5 Conditional Pseudospectrum . . . . . . . . . . . 123

3.6 Structured Pseudospectra . . . . . . . . . . . . . . . 124

3.6.1 Structured Pseudospectrum . . . . . . . . . . . . 124

3.6.2 The Structured Essential Pseudospectra . . . . . . 125

3.6.3 The Structured $S$-Pseudospectra . . . . . . . . 126

3.6.4 The Structured $S$-Essential Pseudospectra . . . . . 128

4 Perturbation of Unbounded Linear Operators by $\gamma$-Relative Boundedness 129

4.1 Sum of Closable Operators . . . . . . . . . . . . . . . . . 129

4.1 .1 Norm Operators . . . . . . . . . . . . . . . . . . . 129

4.1.2 Kuratowski Measure of Noncompactness . . . . . 135

4.2 Block Operator Matrices . . . . . . . . . . . . . . . . . 137 
4.2.1 $2 \times 2$ Block Operator Matrices . . . . . . . . . . 137

4.2.2 $3 \times 3$ Block Operator Matrices . . . . . . . . . . 139

5 Essential Spectra 143

5.1 Characterization of Essential Spectra . . . . . . . . 143

5.1.1 Characterization of Left and Right Weyl Essential Spectra . . . . . . . . . . . . 143

5.1.2 Characterization of Left and Right Jeribi Essential Spectra . . . . . . . . . . . . . 149

5.2 Stability of Essential Approximate Point Spectrum and Essential Defect Spectrum of Linear Operator . . . . . . 156

5.2.1 Stability of Essential Spectra . . . . . . . . . . 156

5.2.2 Invariance of Essential Spectra . . . . . . . . . 159

5.3 Convergence . . . . . . . . . . . . . 162

5.3.1 Convergence Compactly . . . . . . . . . . . 162

5.3.2 Convergence in the Generalized Sense . . . . . . 169

$6 S$-Essential Spectra of Closed Linear Operator on a Banach Space $\quad 183$

$6.1 \quad S$-Essential Spectra . . . . . . . . . . . . . 183

6.1.1 Characterization of $S$-Essential Spectra . . . . . 183

6.1.2 Stability of $S$-Essential Spectra of Closed Linear Operator . . . . . . . . . . . . 188

$6.2 S$-Left and $S$-Right Essential Spectra . . . . . . . . . 195

6.2.1 Stability of $S$-Left and $S$-Right Fredholm Spectra . 195

6.2.2 Stability of $S$-Left and $S$-Right Browder Spectra . . 201

$7 \quad S$-Essential Spectrum and Measure of Non-Strict-Singularity 205

7.1 A Characterization of the $S$-Essential Spectrum . . . . . 205

7.2 The $S$-Essential Spectra of $2 \times 2$ Block Operator Matrices 210

$8 \quad S$-Pseudospectra and Structured $S$-Pseudospectra 217

8.1 Study of the $S$-Pseudospectra . . . . . . . . . . . 217

8.2 Characterization of the Structured $S$-Pseudospectra . . . 223 
8.3 Characterization of the Structured $S$-Essential Pseudospectra ..................... 231

\section{Structured Essential Pseudospectra}

9.1 On a Characterization of the Structured Wolf, AmmarJeribi, and Browder Essential Pseudospectra . . . . . . . 239

9.1.1 Structured Ammar-Jeribi, and Browder Essential Pseudospectra . . . . . . . . . . . . . . . . . 239

9.1.2 A Characterization of the Structured Browder Essential Pseudospectrum . . . . . . . . . . . 252

9.2 Some Description of the Structured Essential Pseudospectra . . . . . . . . . . . . . . 262

9.2.1 Relationship Between Structured Jeribi and Structured Ammar-Jeribi Essential Pseudospectra . . . . 262

9.2.2 A Characterization of the Structured AmmarJeribi Essential Pseudospectrum . . . . . . . . . . 264

10 Structured Essential Pseudospectra and Measure of Noncompactness

10.1 New Description of the Structured Essential Pseudospectra 269

10.1.1 A Characterization of the Structured AmmarJeribi Essential Pseudospectrum by Kuratowski Measure of Noncompactness . . . . . . . . . . . . 269

10.1.2 A Characterization of the Structured Browder Essential Pseudospectrum by Means of Measure of Non-Strict-Singularity . . . . . . . . . . . . 274

11 A Characterization of the Essential Pseudospectra 279

11.1 Approximation of $\varepsilon$-Pseudospectrum . . . . . . . . . . 279

11.2 A Characterization of Approximation Pseudospectrum . . 283

11.3 Essential Approximation Pseudospectrum . . . . . . . 293

11.4 Properties of Essential Pseudospectra ． . . . . . . . . . . 298

11.5 Pseudospectrum of Block Operator Matrices . . . . . . 305 
12 Conditional Pseudospectra 315

12.1 Some Properties of $\Sigma^{\varepsilon}(A) \quad \ldots \ldots \ldots$

12.2 Characterization of Condition Pseudospectrum . . . . . 322

Bibliography

Index 


\section{About the Author}

\section{Aref Jeribi, PhD}

Professor, Department of Mathematics, University of Sfax, Tunisia

Aref Jeribi, PhD, is Professor in the Department of Mathematics at the University of Sfax, Tunisia. He is the author of the book Spectral Theory and Applications of Linear Operators and Block Operator Matrices (2015) and co-author of the book Nonlinear Functional Analysis in Banach Spaces and Banach Algebras: Fixed Point Theory under Weak Topology for Nonlinear Operators and Block Operator Matrices with Applications (CRC Press, 2015). He has published many journal articles in international journals. His areas of interest include spectral theory, matrice operators, transport theory, Gribov operator, Bargman space, fixed point theory, Riesz basis, and linear relations. 
$\Longrightarrow$ Taylor \& Francis

Taylor \& Francis Group

http://taylorandfrancis.com 


\section{Preface}

This book is intended to provide a fairly comprehensive study of spectral theory of linear operators defined on Banach spaces. The central items of interest include various essential spectra, but we also consider some of their generalizations that have been studied in recent years.

As the spectral theory of operators is an important part of functional analysis and has numerous applications in many parts of mathematics and, we require, in this book, some modest prerequisites from functional analysis and operator theory are necessary that the reader can find in the classical texts of functional analysis. We therefore have the hope that this book is accessible to newcomers and graduate students of mathematics with a standard background in analysis.

A considerable part of the content of this book corresponds to research activities developed in collaboration with some colleagues as well as with some of my graduate students over the course of several years. This book is considered as an attempt to organize the available material, most of which exists only in the form of research papers scattered throughout the literature. For this reason, it has been a great pleasure for me to organize the material in this book.

In recent years, spectral theory has witnessed an explosive development. In this book, we present a survey of results concerning various types of essential spectra and pseudospectra in a unified, axiomatic way, and we also gathered several topics that are new but that relate only to those concepts and methods emanating from other parts of the book.

The book covers an important list of topics in spectral theory and will be an excellent choice. It is well written, giving a survey of results 
concerning various types of spectra in a unified, axiomatic way. The main topics include essential spectra, essential pseudospectra, structured essential pseudospectra, and their relative sets.

We do hope that this book will be very useful for researchers, since it represents not only a collection of a previously heterogeneous material but is also an innovation through several extensions.

Of course, it is impossible for a single book to cover such a huge field of research. In making personal choices for inclusion of material, we tried to give useful complementary references in this research area, hence probably neglecting some relevant works. We would be very grateful to receive any comments from readers and researchers, providing us with some information concerning some missing references.

We would like to thank Salma Charfi for the improvement she has made in the introduction of this book. We are indebted to her. We would also like to thank Aymen Ammar for the improvement he has made throughout this book, and we are very grateful to him. Moreover, we should mention that the thesis work, performed under my direction, by my former students and present colleagues Asrar Elleuch, Mohamemd Zerai Dhahri, Bilel Boukettaya, Kamel Mahfoudhi, and Faten Bouzayeni, obtained results that have helped us in writing this book. Finally, we apologize in case we have forgotten to quote any author who has contributed, directly or indirectly, to this work. 


\section{Chapter 1}

\section{Introduction}

Recently, the interest in description of essential spectra remains high because of the abundance of practical applications that help scientists to deal with information overload. This book is intended to provide an important list of topics in spectral theory of linear operators defined on Banach spaces. Central items of interest include Fredholm operators and various characterizations of essential spectra.

In this book, a survey of the state of the art of research related to essential spectra of closed, densely defined, and linear operators subjected to various perturbations is outlined. As important supersets of the essential spectra, the description of essential pseudospectra in this book are interesting objects by themselves, since they carry more information and have a better convergence and approximation properties than essential spectra. We are also interested in giving a much better insight into the essential pseudospectra, by studying some sets called structured essential pseudospectra. Further, a significant amount of research has been done in this book to treat important characterizations of $S$-essential spectra and $S$ structured essential pseudospectra.

In this book, we also turn our attention to the important concept of condition pseudospectrum which carries more information than spectrum and pseudospectrum, especially, about transient instead of just asymptotic behavior of dynamical systems. 
Now, let us describe its contents in the following sections.

\subsection{ESSENTIAL SPECTRA AND RELATIVE ESSENTIAL SPECTRA}

The theory of the essential spectra of linear operators in Banach spaces is a modern section of the spectral analysis. It has numerous applications in many parts of mathematics and physics including matrix theory, function theory, complex analysis, differential and integral equations, and control theory.

The original definition of the essential spectrum goes back to $\mathrm{H}$. Weyl [158] around 1909, when he defined the essential spectrum for a self-adjoint operator $A$ on a Hilbert space as the set of all points of the spectrum of $A$ that are not isolated eigenvalues of finite algebraic multiplicity and he proved that the addition of a compact operator to $A$ does not affect the essential spectrum. Irrespective of whether $A$ is bounded or not on a Banach space $X$, there are many ways to define the essential spectrum, most of them are enlargement of the continuous spectrum. Hence, we can find several definitions of the essential spectrum in the literature, which coincide for self-adjoint operators on Hilbert spaces (see, for example, [79, 137]).

On the other hand, the concept of essential spectra was introduced and studied by many mathematicians and we can cite the contributions of $\mathrm{H}$. Weyl and his collaborators (see, for instance [1-3, 48, 82, 83, 108, 120, 141, 154, 158]). Further important characterizations concerning essential spectra and their applications to transport operators are established by A. Jeribi and his collaborators (see Refs. [1,4-9, 11-14, 19, 26, 31, 34, 36, 37, $40,41,43-47,51,53,59,60,84-88,90,94,95,97-100,114-116,123])$.

Among these essential spectra, the following sets are defined for a closed densely defined linear operator $A$ : 


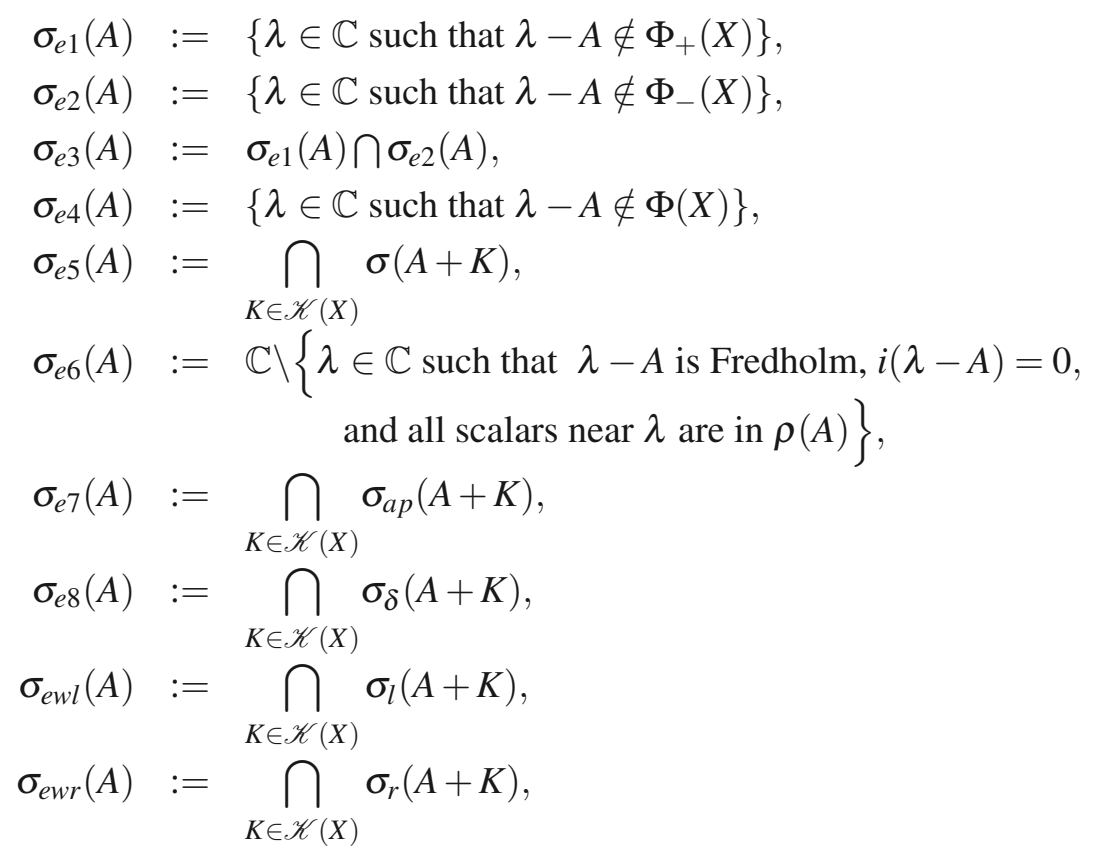

where $\Phi_{+}(X), \Phi_{-}(X)$, and $\Phi(X)$ denote the sets of upper semi-Fredholm, lower semi-Fredholm, and Fredholm operators, respectively; $\rho(A), \sigma(A)$, and $i(A)$ denote the resolvent set, the spectrum, and the index of $A$, respectively; $\mathscr{K}(X)$ denotes the set of all compact linear operators on $X$, and

$$
\begin{aligned}
\sigma_{a p}(A) & :=\left\{\lambda \in \mathbb{C} \text { such that } \inf _{\|x\|=1, x \in \mathscr{D}(A)}\|(\lambda-A) x\|=0\right\} \\
\sigma_{\delta}(A) & :=\{\lambda \in \mathbb{C} \text { such that } \lambda-A \text { is not surjective }\} \\
\sigma_{l}(A) & :=\{\lambda \in \mathbb{C} \text { such that } \lambda-A \text { is not left invertible }\} \\
\sigma_{r}(A) & :=\{\lambda \in \mathbb{C} \text { such that } \lambda-A \text { is not right invertible }\} .
\end{aligned}
$$

The sets $\sigma_{e 1}(\cdot)$ and $\sigma_{e 2}(\cdot)$ are the Gustafson and Weidmann essential spectra, respectively [79]; $\sigma_{e 3}(\cdot)$ is the Kato essential spectrum [108]; the subset $\sigma_{e 4}(\cdot)$ is the Wolf essential spectrum [160]; $\sigma_{e 5}(\cdot)$ is the Schechter essential spectrum [86-88]; and $\sigma_{e 6}(\cdot)$ denotes the Browder essential spectrum $[48,96]$. The subset $\sigma_{e 7}(\cdot)$ was introduced by V. Racočević [133] and designates the essential approximate point spectrum, and $\sigma_{e 8}(\cdot)$ is 
the essential defect spectrum and was introduced by C. Shmoeger [143]. $\sigma_{\text {ewl }}(\cdot)$ and $\sigma_{\text {ewr }}(\cdot)$ are the left and the right of Weyl essential spectra, respectively.

Considerable attention has also been devoted by A. Jeribi $[1,93,113]$ to give a new characterization of the Schechter essential spectrum named the Jeribi essential spectrum, $\sigma_{j}(A)$, of $A \in \mathscr{C}(X)$ (the set of closed densely defined linear operators on $X$ ) defined by

$$
\sigma_{j}(A)=\bigcap_{K \in \mathscr{W}^{*}(X)} \sigma(A+K)
$$

where $\mathscr{W}^{*}(X)$ stands for each one of the sets $\mathscr{W}(X)$ (the set of weakly compact operators) or $\mathscr{S}(X)$ (the set of strictly singular operators).

Recently, an important progress has been made by A. Ammar, B. Boukettaya, and A. Jeribi [20], who were interested in studying the stability problem of the left and right Weyl operator sets; and they defined the left and right Jeribi essential spectra for a closed densely defined operator $A$ as:

$$
\sigma_{j}^{l}(A)=\bigcap_{K \in \mathscr{W}^{*}(X)} \sigma_{l}(A+K)
$$

and

$$
\sigma_{j}^{r}(A)=\bigcap_{K \in \mathscr{W}^{*}(X)} \sigma_{r}(A+K) .
$$

At the first sight, $\sigma_{e w l}(A)\left(\right.$ resp. $\left.\sigma_{e w r}(A)\right)$ and $\sigma_{j}^{l}(A)\left(\right.$ resp. $\left.\sigma_{j}^{r}(A)\right)$ seem to be not equal. However, the authors has proved that $\sigma_{e w l}(A)=\sigma_{j}^{l}(A)$ and $\sigma_{e w r}(A)=\sigma_{j}^{r}(A)$ in $L_{1}$-spaces or $X$ satisfies the Dunford-Pettis property; and hence $\sigma_{\text {ewl }}(A)$ and $\sigma_{e w r}(A)$ may be viewed as an extension of $\sigma_{j}^{l}(A)$ and $\sigma_{j}^{r}(A)$, respectively.

On the other hand, the question of stability of the left and the right essential Weyl spectra was well-treated in Ref. [20] by using the concept of polynomially Riesz operators in order to give a refinement on the definition of these essential spectra via this concept and to show that compactness condition can be relaxed in a very general Banach space setting. 
Motivated by the notion of measure of noncompactness, A. Ammar, M. Z. Dhahri and A. Jeribi [23] are interested in the description of the essential approximate point spectrum and the essential defect spectrum of a closed densely defined linear operator by means of upper semi-Fredholm and lower semi-Fredholm operators, respectively.

In literature, the spectral theory of linear operators have been enriched by S. Goldberg [75], who presented a development of some powerful methods for the study of the convergence of a sequence of linear operators in a Banach space and the investigation of the convergence compactly to zero. Recently, A. Ammar and A. Jeribi are concerned in work [32], not only with the case of bounded linear operators $\left(A_{n}\right)_{n}$ converging compactly to a bounded operator $A$, but also with the case of a sequence of closed linear operators $\left(A_{n}\right)_{n}$ converging in the generalized sense to a closed linear operator $A$.

The notion of generalized convergence was approached as a generalization of convergence in norm for possibly unbounded linear operators, as well as a reliable method for comparing operators. Further, this notion make the authors generalize the results of T. Kato [108], which essentially represents the convergence between their graphs in a certain distance. The obtained results were exploited in Ref. [27] to examine the relationship between the various essential spectrum of $A_{n}$ and $A$, where $\left(A_{n}\right)_{n}$ converges in the generalized sense to $A$.

In the last years, there have been many studies of the operators pencils, $\lambda S-A, \lambda \in \mathbb{C}$ (operator-valued functions of a complex argument) (see, for example, $[119,147])$. It is known that many problems of mathematical physics (e.g., quantum theory, transport theory, $\cdots$ ) are reduced to the study of the essential spectra of $\lambda S-A$. For this, it seems interesting to study the following $S$-essential spectra: 


$$
\begin{aligned}
\sigma_{e 1, S}(A):=\left\{\lambda \in \mathbb{C} \text { such that } \lambda S-A \notin \Phi_{+}(X)\right\}, \\
\sigma_{e 2, S}(A):=\left\{\lambda \in \mathbb{C} \text { such that } \lambda S-A \notin \Phi_{-}(X)\right\}, \\
\sigma_{e 3, S}(A):=\left\{\lambda \in \mathbb{C} \text { such that } \lambda S-A \notin \Phi_{+}(X) \cup \Phi_{-}(X)\right\}, \\
\sigma_{e 4, S}(A):=\{\lambda \in \mathbb{C} \text { such that } \lambda S-A \notin \Phi(X)\}, \\
\sigma_{e 5, S}(A):=\{\lambda \in \mathbb{C} \text { such that } \lambda S-A \notin \mathscr{W}(X)\}, \\
\sigma_{b, S}(A):=\{\lambda \in \mathbb{C} \text { such that } \lambda S-A \notin \mathscr{B}(X)\}, \\
\sigma_{e l, S}(A):=\left\{\lambda \in \mathbb{C} \text { such that } \lambda S-A \notin \Phi_{l}(X)\right\}, \\
\sigma_{e r, S}(A):=\left\{\lambda \in \mathbb{C} \text { such that } \lambda S-A \notin \Phi_{r}(X)\right\}, \\
\sigma_{e w l, S}(A):=\left\{\lambda \in \mathbb{C} \text { such that } \lambda S-A \notin \mathscr{W}_{l}(X)\right\}, \\
\sigma_{e w r, S}(A):=\left\{\lambda \in \mathbb{C} \text { such that } \lambda S-A \notin \mathscr{W}_{r}(X)\right\}, \\
\sigma_{b l, S}(A):=\left\{\lambda \in \mathbb{C} \text { such that } \lambda S-A \notin \mathscr{B}^{l}(X)\right\}, \\
\sigma_{b r, S}(A):=\left\{\lambda \in \mathbb{C} \text { such that } \lambda S-A \notin \mathscr{B}^{r}(X)\right\}, \\
\sigma_{e a p, S}(A):=\left\{\lambda \in \mathbb{C} \text { such that } \lambda S-A \notin \Phi_{+}(X) \text { and } i(\lambda S-A) \leq 0\right\}, \\
\sigma_{e \delta, S}(A):=\left\{\lambda \in \mathbb{C} \text { such that } \lambda S-A \notin \Phi_{-}(X) \text { and } i(\lambda S-A) \geq 0\right\},
\end{aligned}
$$

where $\mathscr{B}(X)$ is the set of Riesz-Schauder operators; $\Phi_{l}(X)$ is the set of left Fredholm operators; $\Phi_{r}(X)$ is the set of right Fredholm operators; $\mathscr{W}_{l}(X)$ is the set of left Weyl operators; $\mathscr{W}_{r}(X)$ is the set of right Weyl operators; $\mathscr{B}^{l}(X)$ is the set of left Browder operators; and $\mathscr{B}^{r}(X)$ is the set of right Browder operators. They can be ordered as

$$
\begin{gathered}
\sigma_{e 3, S}(A)=\sigma_{e 1, S}(A) \bigcap \sigma_{e 2, S}(A) \subseteq \sigma_{e 4, S}(A) \subseteq \sigma_{e 5, S}(A) \subseteq \sigma_{b, S}(A), \\
\sigma_{e l, S}(A) \subseteq \sigma_{e w l, S}(A) \subseteq \sigma_{b l, S}(A), \\
\sigma_{e r, S}(A) \subseteq \sigma_{e w r, S}(A) \subseteq \sigma_{b r, S}(A) .
\end{gathered}
$$

In Ref. [29], the authors proved that

$\sigma_{e 4, S}(A)=\sigma_{e l, S}(A) \bigcup \sigma_{e r, S}(A), \sigma_{e 1, S}(A) \subset \sigma_{e l, S}(A)$ and $\sigma_{e 2, S}(A) \subset \sigma_{e r, S}(A)$.

Note that if $S=I$, we recover the usual definition of the essential spectra of a bounded linear operator $A$ defined in the first section of this introduction. These relative essential spectra drew the attention of Jeribi and his collaborators in Refs. [1, 10, 20, 29, 101]. 
More precisely, the authors A. Ammar, B. Boukettaya, and A. Jeribi pursued the analysis started in Refs. $[1,163,164]$ for $S$-left-right essential spectra and they studied the invariance of $\sigma_{e i, S}(\cdot)(i=l, r, w l, w r)$ by some class of perturbations and extended a part of the results obtained in Ref. [98] to a large class of perturbation operators $\mathscr{R}(X)$, which contains $\mathscr{F}(X)$ for the $S$-left and $S$-right spectrum, where $\mathscr{R}(X)$ (resp. $\mathscr{F}(X))$ denotes the set of Riesz operators (resp. Fredholm perturbations). They applied their obtained results to describe the $S$-essential spectra of an integro-differential operator with abstract boundary conditions acting in the Banach space.

At the same time, an important progress has been made in order to describe various relative essential spectra and we can cite in this context the contribution of the work of A. Ammar, M. Z. Dhahri and A. Jeribi in Ref. [25], where the authors are inspired by the work [10] and studied some types of $S$-essential spectra of linear bounded operators on a Banach space $X$. More precisely, in Ref. [25], we find a detailed treatment of some subsets of $S$-essential spectra of closed linear operators by means of the measure of non-strict-singularity.

When dealing with block operator matrices, we recall that the papers $[39,52,54-56,103,111,120,146]$ are concerned with the study of the $\mathscr{I}$ essential spectra of operators defined by a $2 \times 2$ block operator matrix that

$$
\mathscr{L}_{0}=\left(\begin{array}{cc}
A & B \\
C & D
\end{array}\right)
$$

acts on the product $X \times Y$ of Banach spaces, where $\mathscr{I}$ is the identity operator defined on the product space $X \times Y$ by

$$
\mathscr{I}=\left(\begin{array}{cc}
I & 0 \\
0 & I
\end{array}\right) .
$$

Inspired by the works $[39,111,120,146]$, the aim of the authors in Ref. [101] was to generalize the previous work and they considered a bounded operator $S$ formally defined on the product Banach space $X \times Y$ as

$$
S=\left(\begin{array}{ll}
M_{1} & M_{2} \\
M_{3} & M_{4}
\end{array}\right) \text {. }
$$


A considerable attention has been also devoted in Ref. [24] in order to give the characterization of the $S$-essential spectra of the $2 \times 2$ matrix operator $\mathscr{L}_{0}$ acting on a Banach space are given by using the notion of measure of non-strict-singularity. These results are considered as generalizations of the paper of N. Moalla in Ref. [122], where $S$-essential spectra of some $2 \times 2$ operator matrices on $X \times X$ are discussed with $S=I$.

\subsection{ESSENTIAL PSEUDOSPECTRA}

In 1967, J. M. Varah [155] introduced the first idea of pseudospectra. In 1986, J. H. Wilkinson [159] came up with the modern interpretation of pseudospectrum where he defined it for an arbitrary matrix norm induced by a vector norm. Throughout the 1990s, L. N. Trefethen [135, 150-152] not only initiated the study of pseudospectrum for matrices and operators, but also he talked of approximate eigenvalues and pseudospectrum and used this notion to study interesting problems in mathematical physics. By the same token, several authors worked on this field. For example, we may refer to E. B. Davies [58], A. Harrabi [80], A. Jeribi [1], E. Shargorodsky [145], and M. P. H. Wolff [161] who had introduced the term approximation pseudospectrum for linear operators.

Pseudospectra are interesting objects by themselves since they carry more information than spectra, especially about transient instead of just asymptotic behavior of dynamical systems. Also, they have better convergence and approximation properties than spectra. The definition of pseudospectra of a closed densely defined linear operator $A$, for every $\varepsilon>0$, is given by:

$$
\sigma_{\varepsilon}(A):=\sigma(A) \bigcup\left\{\lambda \in \mathbb{C} \text { such that }\left\|(\lambda-A)^{-1}\right\|>\frac{1}{\varepsilon}\right\},
$$


or by

$$
\Sigma_{\varepsilon}(A):=\sigma(A) \bigcup\left\{\lambda \in \mathbb{C} \text { such that }\left\|(\lambda-A)^{-1}\right\| \geq \frac{1}{\varepsilon}\right\} .
$$

By convention, we write $\left\|(\lambda-A)^{-1}\right\|=\infty$ if $\lambda \in \sigma(A)$, (spectrum of $A$ ). For $\varepsilon>0$, it can be shown that $\sigma_{\varepsilon}(A)$ is a larger set and is never empty. The pseudospectra of $A$ are a family of strictly nested closed sets, which grow to fill the whole complex plane as $\varepsilon \rightarrow \infty$ (see [80,151,152]). From these definitions, it follows that the pseudospectra associated with various $\varepsilon$ are nested sets. Then, for all $0<\varepsilon_{1}<\varepsilon_{2}$, we have

$$
\sigma(A) \subset \sigma_{\varepsilon_{1}}(A) \subset \sigma_{\varepsilon_{2}}(A) \text { and } \sigma(A) \subset \Sigma_{\varepsilon_{1}}(A) \subset \Sigma_{\varepsilon_{2}}(A),
$$

and that the intersections of all the pseudospectra are the spectrum,

$$
\bigcap_{\varepsilon>0} \sigma_{\varepsilon}(A)=\sigma(A)=\bigcap_{\varepsilon>0} \Sigma_{\varepsilon}(A)
$$

In [58], E. B. Davies has defined another equivalent of pseudospectrum. One is in terms of perturbations of the spectrum, that is, for any closed operator $A$, we have

$$
\sigma_{\varepsilon}(A):=\bigcup_{\|D\|<\varepsilon} \sigma(A+D)
$$

Inspired by the notion of pseudospectra, A. Ammar and A. Jeribi in their works [29], thought to extend these results for essential spectra of densely closed linear operators on a Banach space. They declared the new concept of the essential pseudospectra of densely closed linear operators on a Banach space. Because of the existence of several essential spectra, they were interested in focusing their study on the pseudo-Browder essential spectrum. This set was shown to be characterized in the way one would expect by analogy with essential numerical range. As a consequence, the authors in Ref. [29] located the pseudo-Browder essential spectrum between the essential spectra and the essential numerical range. 
More precisely, for $A \in \mathscr{C}(X)$ and for every $\varepsilon>0$, they defined the pseudoBrowder essential spectrum as follows:

$$
\sigma_{e 6, \varepsilon}(A)=\sigma_{e 6}(A) \bigcup\left\{\lambda \in \mathbb{C} \text { such that }\left\|R_{b}(A, \lambda)\right\|>\frac{1}{\varepsilon}\right\},
$$

where $R_{b}(A, \lambda)$ is the Browder resolvent of $A$. The aim of this concept is to study the existence of eigenvalues far from the Browder essential spectrum and also to search the instability of the Browder essential spectrum under every perturbation. Their study of pseudo-Browder essential spectrum enabled them to determine and localize the Browder essential spectrum of a closed, densely defined linear operator on a Banach space.

In Refs. [1, 21, 29, 30], A. Jeribi and his collaborators pursued their studies about essential pseudospectra and defined the following sets:

$$
\begin{aligned}
\sigma_{e 1, \varepsilon}(A):= & \left\{\lambda \in \mathbb{C} \text { such that } \lambda-A \notin \Phi_{+}^{\varepsilon}(X)\right\}, \\
\sigma_{e 2, \varepsilon}(A):= & \left.:=\in \mathbb{C} \text { such that } \lambda-A \notin \Phi_{-}^{\varepsilon}(X)\right\}, \\
\sigma_{e 3, \varepsilon}(A):= & \left.: \lambda \in \mathbb{C} \text { such that } \lambda-A \notin \Phi_{ \pm}^{\varepsilon}(X)\right\}, \\
\sigma_{e 4, \varepsilon}(A):= & \left\{\lambda \in \mathbb{C} \text { such that } \lambda-A \notin \Phi^{\varepsilon}(X)\right\}, \\
\sigma_{e 5, \varepsilon}(A):=\bigcap_{K \in \mathscr{K}(X)} \sigma_{\varepsilon}(A+K), & \\
\sigma_{e a p, \varepsilon}(A):= & \sigma_{e 1, \varepsilon}(A) \bigcup\{\lambda \in \mathbb{C}: i(\lambda-A-D)>0, \forall\|D\|<\varepsilon\}, \\
\sigma_{e \delta, \varepsilon}(A):= & \sigma_{e 2, \varepsilon}(A) \bigcup\{\lambda \in \mathbb{C}: i(\lambda-A-D)<0, \forall\|D\|<\varepsilon\},
\end{aligned}
$$

where $\Phi_{+}^{\varepsilon}(X)$ (resp. $\left.\Phi_{-}^{\varepsilon}(X)\right)$ denotes the set of upper (resp. lower) pseudo semi-Fredholm operator, $\Phi_{ \pm}^{\varepsilon}(X)$ denotes the set of pseudo semi-Fredholm operator, and $\Phi^{\varepsilon}(X)$ denotes the set of pseudo Fredholm operators. Note that if $\varepsilon$ tends to 0 , we recover the usual definition of the essential spectra of a closed linear operator $A$ defined in the first section of this introduction.

In [161], M. P. H. Wolff has given a motivation to study the essential approximation pseudospectrum. In [33], A. Ammar, A. Jeribi and K. Mahfoudhi showed that the notion of essential approximation pseudospectrum can be extended by devoting our studies to the essential approxima- 
tion spectrum. For $\varepsilon>0$ and $A \in \mathscr{C}(X)$, they define

$$
\begin{aligned}
\sigma_{\text {eap }, \varepsilon}(A) & =\bigcap_{K \in \mathscr{K}(X)} \sigma_{a p, \varepsilon}(A+K), \\
\Sigma_{\text {eap }, \varepsilon}(A) & =\bigcap_{K \in \mathscr{K}(X)} \Sigma_{a p, \varepsilon}(A+K),
\end{aligned}
$$

where

$$
\sigma_{a p, \varepsilon}(A):=\left\{\lambda \in \mathbb{C} \text { such that } \inf _{x \in \mathscr{D}(A),\|x\|=1}\|(\lambda-A) x\|<\varepsilon\right\},
$$

and

$$
\Sigma_{a p, \varepsilon}(A):=\left\{\lambda \in \mathbb{C} \text { such that } \inf _{x \in \mathscr{D}(A),\|x\|=1}\|(\lambda-A) x\| \leq \varepsilon\right\} .
$$

In their work, the authors measure the sensitivity of the set $\sigma_{a p}(A)$ with respect to additive perturbations of $A$ by an operator $D \in \mathscr{L}(X)$ of a norm less than $\varepsilon$. So, they define the approximation pseudospectrum of $A$ by

$$
\sigma_{a p, \varepsilon}(A)=\bigcup_{\|D\|<\varepsilon} \sigma_{a p}(A+D),
$$

and they give a characterization of the essential approximation pseudospectrum $\sigma_{e a p, \varepsilon}(\cdot)$ which nicely blends these properties of the essential and the approximation pseudospectra, and accordingly the authors are interested in the following essential approximation spectrum

$$
\sigma_{\text {eap }}(A):=\bigcap_{K \in \mathscr{K}(X)} \sigma_{a p}(A+K) .
$$

As a consequence, the authors obtain the following result

$$
\sigma_{\text {eap }, \varepsilon}(A)=\bigcup_{\|D\|<\varepsilon} \sigma_{\text {eap }}(A+D) .
$$




\subsection{STRUCTURED ESSENTIAL PSEUDOSPECTRA AND RELATIVE STRUCTURED ESSENTIAL PSEUDOSPECTRA}

Structured pseudospectra are very useful tools in control theory and other fields [81]. In particular, D. Hinrichsen and B. Kelb [81] presented in 1993 a graphical method to determine and visualize the spectral value sets of a matrix $A$.

We also refer the reader to E. B. Davies [58] who defined the structured pseudospectra, or spectral value sets of a closed densely defined linear operator $A$ on a Banach space $X$ by

$$
\sigma(A, B, C, \varepsilon)=\bigcup_{\|D\|<\varepsilon} \sigma(A+C D B)
$$

where $B \in \mathscr{L}(X, Y)$ (the set of bounded linear operators from $X$ into $Y$ ) and $C \in \mathscr{L}(Z, X)$.

The notion of structured pseudospectra drew the attention of A. Elleuch and A. Jeribi who have recently been concerned in $[67,68]$ with the study of essential spectra of an operator $A$ which is subjected to structured perturbation of the form $A \rightsquigarrow A+C D B$, where $B, C$ are given bounded operators and $D$ is unknown disturbance operator satisfies $\|D\|<\varepsilon$ for a given $\varepsilon>0$. More specifically, inspired by [1, 86-89, 91, 112], where the authors proved the invariance of the Schechter essential spectrum of a closed densely defined linear operators, A. Elleuch and A. Jeribi, in $[67,68]$, extended the obtained results for the case of structured AmmarJeribi essential pseudospectrum. For this analysis, they needed to characterize the structured pseudospectra of bounded operator and they defined the following structured essential pseudospectra of a closed, densely defined linear operator $A$ with respect to the perturbation structure $(B, C)$, 
where $B \in \mathscr{L}(X, Y), C \in \mathscr{L}(Z, X)$ and uncertainty level $\varepsilon>0$,

$$
\begin{aligned}
& \sigma_{e 4}(A, B, C, \varepsilon):=\bigcup_{\|D\|<\varepsilon} \sigma_{e 4}(A+C D B), \\
& \sigma_{e 5}(A, B, C, \varepsilon):=\bigcup_{\|D\|<\varepsilon} \sigma_{e 5}(A+C D B), \\
& \sigma_{e 6}(A, B, C, \varepsilon):=\bigcup_{\|D\|<\varepsilon} \sigma_{e 6}(A+C D B) .
\end{aligned}
$$

The subset $\sigma_{e 4}(A, B, C, \varepsilon)$ is the structured Wolf essential pseudospectrum, $\sigma_{e 5}(A, B, C, \varepsilon)$ is the structured Ammar-Jeribi essential pseudospectrum, and $\sigma_{e 6}(A, B, C, \varepsilon)$ denotes the structured Browder essential pseudospectrum.

The main purpose of the authors in Ref. [67] consists in studying the structured essential pseudospectra of the sum of two operators in order to improve and extend the results of Refs. $[1,12,144]$ which were done for various essential spectra. In addition, the authors described the behavior of structured Ammar-Jeribi essential pseudospectrum and they presented a fine description of this set by means of compact and Fredholm perturbations.

Further, they used in Ref. [68] some criteria in order to prove the stability of the structured Wolf and the structured Ammar-Jeribi essential pseudospectra. They also used the notion of the measure of noncompactness to give a new characterization of the structured Ammar-Jeribi essential pseudospectrum and they presented some properties of the structured Ammar-Jeribi and Browder essential pseudospectra by means of the measure of non-strict-singularity.

On the other hand, based on the definition of the $S$-essential spectra and the structured $S$-pseudospectra, A. Elleuch and A. Jeribi, in [67, 68], introduced and characterized the structured $S$-pseudospectrum of a closed, densely defined linear operator on a Banach space $X$. They also established 
a relationship between the structured $S$-essential pseudospectra and the $S$ essential spectra of a bounded linear operator under some conditions.

\subsection{CONDITION PSEUDOSPECTRUM}

There are several generalizations of the concept of the spectrum in literature such as Ransford spectrum [134], pseudospectrum [1, 80, 152], and condition spectrum [109]. It is natural to ask whether there are any results similar for operator in infinite dimensional Banach spaces for these sets. The concept of condition pseudospectrum is very important since it carries more information than spectra and pseudospectra, especially, about transient instead of just asymptotic behavior of dynamical systems. Also, condition pseudospectrums have better convergence and approximation properties than spectra and pseudospectra. For a detailed study of the properties of the condition spectrum in finite dimensional space or in Banach algebras, we may refer to S. H. Kulkarni and D. Sukumar in Ref. [109], M. Karow in [106] and G. K. Kumar and S. H. Lui in Ref. [110].

The aim of the authors A. Ammar, A. Jeribi and K. Mahfoudhi in [35] is to show some properties of condition pseudospectra of a linear operator $A$ in Banach spaces and reveal the relation between their condition pseudospectrum and the pseudospectrum (resp. numerical range). One of the central questions consists in characterizing the condition pseudospectra of all bounded linear operators acting in a Banach space. More precisely, the authors consider, in their work, one more such extension in terms of the condition number. In a complex Banach space, the condition pseudospectrum of a bounded linear operator $A$ in infinite dimensional Banach spaces is given for every $0<\varepsilon<1$ by:

$$
\Sigma^{\varepsilon}(A):=\sigma(A) \bigcup\left\{\lambda \in \mathbb{C} \text { such that }\|\lambda-A\|\left\|(\lambda-A)^{-1}\right\|>\frac{1}{\varepsilon}\right\},
$$


with the convention that $\|\lambda-A\|\left\|(\lambda-A)^{-1}\right\|=\infty$, if $\lambda-A$ is not invertible.

The authors summarize some properties and useful results of the condition pseudospectrum and they characterize it in order to improve lots recent results of Refs. $[106,109,110]$ in infinite dimensional Banach spaces.

\subsection{OUTLINE OF CONTENTS}

Going on to a brief indication of the contents of the individual chapters, we mention, first of all that our book consists of 12 chapters.

We recall in Chapter 2 some definitions, notations, and basic information on both bounded and unbounded Fredholm operators. We also introduce the concept of semigroup theory, compactly convergence, measure of noncompactness, gap topology, and quasi-inverse operator. Basic concepts and classical results are summarized in the first two sections. In the subsequent sections we study the approximate point spectrum, which is one of the most important examples of a spectrum in Banach algebras. The approximate point spectrum is closely related with the notions of removable and non-removable ideals.

We continue Chapter 3 by giving some fundamentals about characterization of various spectra and essential spectra such as left and right Jeribi essential spectra, $S$-resolvent set, $S$-spectra, $S$-essential spectra, pseudospectra and structured pseudospectra.

The aim of Chapter 4 is to investigate the perturbation of unbounded linear operators via the concept of $\gamma$-relative boundedness introduced in [92]. In fact, this chapter is a survey of the paper [22] where the authors try to generalize the some results of [61] by using the new concept of the gap and the $\gamma$-relative boundedness in order to establish some sufficient conditions imposed to three unbounded linear operators and to obtain the 
closedness of their algebraic sum. The obtained results are useful to study some specific properties of both $2 \times 2$ and $3 \times 3$ block operator matrices.

Chapter 5 addresses the characterization of essential spectra such as left and right Jeribi and Weyl essential spectra. We also study the stability of essential approximate point spectrum and essential defect spectrum of linear operators and we treat the elegant interaction between essential spectra of closed linear operators and the notion of convergence in the generalized sense.

In Chapters 6 and 7, we focus on the study of the stability of $S$-left and $S$-right essential spectra. Moreover, we give the characterization of the Schechter $S$-essential spectrum by means of compact and Fredholm perturbations and also via the concept of measure of non-strict-singularity. Finally, illustrate the obtained results to matrix operator.

We concentrate ourselves exclusively in Chapter 8 on the characterization of $S$-pseudospectra, structured $S$-pseudospectra, and structured $S$ essential pseudospectra. Inspired by the definition of essential spectra, we define and characterize in Chapter 9 the structured Ammar-Jeribi, Browder and Wolf, essential pseudospectra and in particular, we treat the relationship between structured Jeribi and structured Ammar-Jeribi essential pseudospectra.

Chapter 10 focuses on a new description of the structured essential pseudospectra. Not only do we give a characterization of the structured Ammar-Jeribi essential pseudospectrum by Kuratowski measure of noncompactness but also we investigate the structured Browder essential pseudospectrum by means of measure of non-strict-singularity.

The aim of Chapter 11 is to investigate the essential approximation pseudospectrum of closed, densely defined linear operators on a Banach space. We refine the notion of the $\varepsilon$-pseudospectrum and we focus on the characterization of the approximation pseudospectrum and the essential approximation pseudospectrum. We also establish some properties of essential pseudospectra. Further, we study the pseudospectrum of block operator matrix. 
Introduction

Finally, in Chapter 12, we summarize some properties and useful results of the condition pseudospectrum and we characterize it in infinite dimensional Banach spaces. 


\section{References}

Jeribi, A. , (2015). Spectral Theory and Applications of Linear Operators and Block Operator Matrices, Springer-Verlag, New York.

Jeribi, A. , (2018). Spectral Theory of Linear Non-Self-Adjoint Operators in Hilbert and Banach Spaces: Denseness and Bases with Applications, preprint.

Jeribi, A. , (2018). Denseness, Bases and Frames in Banach Spaces and Applications, de Gruyter, Berlin.

Jeribi, A. , \& Krichen, B. , (2016). Nonlinear Functional Analysis in Banach Spaces and Banach Algebras. Fixed point theory under weak topology for nonlinear operators and block operator matrices with applications. (English) Monographs and Research Notes in

Mathematics. Boca Raton, FL: CRC Press (ISBN 978-1-4987-3388-5/hbk; 978-1-4987-33892/ebook). xvi, 355 p.

Jeribi, A. , Hammami, M. A. , \& Masmoudi, A. , (eds.), (2015). Applied Mathematics in Tunisia. International Conference on Advances in Applied Mathematics (ICAAM), Hammamet, Tunisia, December 1619, 2013. Springer proceedings in mathematics and statistics 131. Cham: Springer (ISBN 978-3-319-18040-3/hbk; 978-3-319-18041-0/ebook). xix, $397 \mathrm{p}$.

Abdelmoumen, B. , Dehici, A. , Jeribi, A. , \& Mnif, M. , (2008). Some new properties of fredholm theory, Schechter essential spectrum, and application to transport theory, J. Inequal. Appl., Art. ID 852676, 1-14.

Abdelmoumen, B. , Jeribi, A. , \& Mnif, M. , (2011). Invariance of the Schechter essential spectrum under polynomially compact operators perturabation, Extr. Math., 26(1), 61-73.

Abdelmoumen, B. , Jeribi, A. , \& Mnif, M. , (2012). On graph measures in banch spaces and description of essential spectra of multidimensional transport equation, Acta Math. Sci. Ser. B Engl. Ed., 32(5), 2050-2064.

Abdelmoumen, B. , Jeribi, A. , \& Mnif, M. , (2012). Measure of weak noncompactness, some new properties in fredholm theory, characterization of the Schechter essential spectrum and application to transport operators, Ricerche Mat., 61, 321-340.

Abdmouleh, F. , Ammar, A. , \& Jeribi, A. , (2013). Stability of the S-Essential Spectra on a Banach Space, Math. Slovaca, 63(2), 299-320.

Abdmouleh, F. , Ammar, A. , \& Jeribi, A. , A (2015). Characterization of the pseudo-browder essential spectra of linear operators and application to a transport equation, J. Comput. Theor. Transp., 44(3), 141-153.

Abdmouleh, F. , Charfi, S. , \& Jeribi, A. , (2012). On a characterization of the essential spectra of the sum and the product of two operators, J. Math. Anal. Appl., 386(1), 83-90.

Abdmouleh, F. , \& Jeribi, A. , (2010). Symmetric family of Fredholm operators of indices zero, stability of essential spectra and application to transport operators, J. Math. Anal. Appl., 364, 414-223.

Abdmouleh, F. , \& Jeribi, A. , (2011). Gustafson, Weidmann, Kato, Wolf, Schechter, Browder, Rako cević and Schmoger essential spectra of the sum of two bounded operators and application to a transport operator, Math. Nachr., 284(2-3), 166-176.

Aiena, P. , (1989). Riesz Operators and Perturbation Ideals, Note Mat., 9(1), 1-27.

Aiena, P. , (2007). Semi-Fredholm Operators, Perturbation Theory and Localized SVEP, Caracas, Venezuela.

Akhmerov, R. R. , Kamenskii, M. , I., Potapov , A. S., Rodkina , A., E., \& Sadovskii, B. N. , (1992). Measures of Noncompactness and Condensing Operators, Birkhäuser Verlag, Basel Boston Berlin.

Alvarez, T. , (1998). Perturbation and coperturbation functions characterizing semi-Fredholm type operators, Math. Proc. R. Ir. Acad., 98 A(1), 41-26.

Ammar, A. , Boukattaya, B. , \& Jeribi, A. , (2014). Stability of the S-left and S-right essential spectra of a linear operator, Acta Math. Scie., Ser. B Engl. Ed., 34(5), 1-13.

Ammar, A. , Boukettaya, B. , \& Jeribi, A. , (2014). Stability of the S-left and S-right essential spectra of a linear operator, Acta Math. Scie. Ser. B Engl. Ed., 34(6), 1922-1934.

Ammar, A. , Boukettaya, B. , \& Jeribi, A. , (2016). A note on the essential pseudospectra and application, Linear and Multilinear Algebra, 64(8), 1474-1483. 
Ammar, A. , Bouzayeni, F. , \& Jeribi, A. , (2017). Perturbation of unbounded linear operators by $\mathrm{y}$-relative boundedness, Ricerche Mat., DOI 10.1007/s11587-017-0341-0, 1-13.

Ammar, A. , Dhahri, M. Z. , \& Jeribi, A. , (2015). Stability of essential approximate point spectrum and essential defect spectrum of linear operator, Filomat, 29(9), 1983-1994.

Ammar, A. , Dhahri, M. Z. , \& Jeribi, A. , (2015). Some properties of the M-essential spectra of closed linear operator on a Banach space, Functional Analysis, Approximation and Computation, 7(1), 15-28.

Ammar, A. , Dhahri, M. Z. , \& Jeribi, A. , (2015). A Characterization of S-essential spectrum by mean of measure of non-strict-singularity and application, Azerbaijan J. Math., V. 5(1), 2218-6816.

Ammar, A. , Dhahri, M. Z. , \& Jeribi, A. , (2015). Some properties of upper triangular $3 \times 3-$ block matrices of linear relations, Boll. Unione Mat. Ital., 8(3), 189-204.

Ammar, A. , Djaidja, N. , \& Jeribi, A. , (2017). The essential spectrum of a sequence of linear operators in Banach spaces, International J. Anal. Appl., 1-7.

Ammar, A. , Heraiz, T. , \& Jeribi, A. , (2017). Essential approximate point spectrum and essential defect spectrum of a sequence of linear operators in Banach spaces, preprint. Ammar, A. , \& Jeribi, A. , (2013). A characterization of the essential pseudospectra on a Banach space, Arab. J. Math., 2(2), 139-145.

Ammar, A. , \& Jeribi, A. , (2013). A characterization of the essential pseudospectra and application to a transport equation, Extr. Math., 28, 95-112.

Ammar, A. , \& Jeribi, A. , (2014). Measures of noncompactness and essential pseudo-spectra on Banach Space, Math. Methods Appli. Sci., 37(3), 447-452.

Ammar, A. , \& Jeribi, A. , (2016). The Weyl essential spectrum of a sequence of linear operators in Banach spaces, Indag. Math. (N. S.), 27(1), 282-295.

Ammar, A. , Jeribi, A. , \& Mahfoudhi, K. , (2017). A characterization of the essential approximation pseudospectrum on a Banach space, Filomat 31(11), 3599-3610.

Ammar, A. , Jeribi, A. , \& Mahfoudhi, K. , (2017). Global bifurcation from the real leading eigenvalue of the transport operator, J. Comput. Theor. Transp., 46(4), 229-241.

Ammar, A. , Jeribi, A. , \& Mahfoudhi, K. , (2017). A Characterization of the Condition Pseudospectrum, preprint.

Ammar, A. , Jeribi, A. , \& Moalla, N. , (2013). On a characterization of the essential spectra of a $3 \times 3$ operator matrix and application to three-group transport operators, Ann. Funct. Anal.

AFA 4(2), 153-170, electronic only.

Ammar, A. , Jeribi, A. , \& Saadaoui, B. , (2017). Frobenius-Schur factorization for multivalued $2 \times 2$ matrices linear operator, Mediterr. J. Math., 14(1), Paper No. 29, 29 p.

Astala, K. , (1980). On measure of noncompactness and ideal variations in Banach spaces, Ann. Acad. Sci. Fenn. Ser. A. I. Math. Diss., 29.

Atkinson, F. V. , Langer, H. , Mennicken, R. , \& Shkalikov, A. A. , (1994). The essential spectrum of some matrix operators, Math. Nachr., 167, 5-20.

Baloudi, H. , \& Jeribi, A. , (2014). Left-Right Fredholm and Weyl spectra of the sum of two bounded operators and application, Mediterr. J. Math., 11, 939-953.

Baloudi, H. , \& Jeribi, A. , (2016). Holomorphically Weyl-decomposably regular. (English) Funct. Anal. Approx. Comput., 8 (2), 13-22.

Banaś, J. , \& Geobel, K. , (1980). Measures of noncompactness in Banach spaces, Lecture Notes in Pure and Applied Mathematics, Volume 60, Marcel Dekker, New York, 259-262.

Ben Ali, N. , Jeribi, A. , \& Moalla, N. , (2010). Essential spectra of some matrix operators, Math. Nachr., 283(9), 1245-1256.

Ben Amar, A. , Jeribi, A. , \& Mnif, M. , (2010). Some applications of the regularity and irreducibility on transport theory, Acta Appl. Math., 110, 431-448.

Ben Amar, A. , Jeribi, A. , \& Krichen, B. , (2010). Essential spectra of a $3 \times 3$ operator matrix and application to three-group transport equation, Inte. Equa. Oper. Theo., 68, 1-21.

Ben Amar, A. , Jeribi, A. , \& Mnif, M. , (2014). Some results on Fredholm and semi-Fredholm operators, Arab. J. Math. (Springer) 3(3), 313-323.

Benharrat, M. , Ammar, A. , Jeribi, A. , \& Messirdi, B. , (2014). On the Kato, semi-regular and essentially semi-regular spectra, Functional Analysis, Approximation and Computation 6(2),

9-22. 
Browder, F. E. , (1961). On the spectral theory of elliptic differential operators, I, Math. Ann., 142, 22-130.

Caradus, S. R. , (1966). Operators of Riesz type, Pacific J. Math., 18, 61-71.

Caradus, S. R. , (1966). Operators with finite ascent and descent, Pacific J. Math., 18, 437-449.

Chaker, W. , Jeribi, A. , \& Krichen, B. , (2015). Demicompact linear operators, essential spectrum and some perturbation results, Math. Nachr., 288(13), 1476-1486.

Charfi, S. , \& Jeribi, A. , (2009). On a characterization of the essential spectra of some matrix operators and applications to two-group transport operators, Math. Z., 262(4), 775-794.

Charfi, S. , Jeribi, A. , \& Moalla, N. , (2013). Time asymptotic behavior of the solution of an abstract Cauchy problem given by a one-velocity transport operator with Maxwell boundary condition, Collectanea Mathematica, 64, 97-109.

Charfi, S. , Jeribi, A. , \& Moalla, R. , (2014). Essential spectra of operator matrices and applications, Methods Appli. Sci. 37(4), 597-608.

Charfi, S. , Jeribi, A. , \& Walha, I. , (2010). Essential Spectra, Matrix Operator and Applications, Acta Appl. Math., 111, (3), 319-337.

Damak, M. , \& Jeribi, A. , (2007). On the essential spectra of some matrix operators and applications, Electron. J. Differential Equations 11, 1-16.

Danes, J. , (1972). On the istratescu measure of noncompactness, Bull. Math. Soc. R. S. Roumanie, 16(64), 403-406.

Davies, E. B. , (2007). Linear Operators and Iheir Spectra, Cambridge University Press, New York.

Dehici, A. , Latrach, K. , \& Jeribi, A. , (2002). On a transport operator arising in growing cell populations I. Spectral analysis, Advances in Mathematics Research, pp. 149-175.

Dehici, A. , Jeribi, A. , \& Latrach, K. , Spectral analysis of a transport operator arising in growing cell populations, Acta Appl. Math., 92(1), 37-62 (2006).

Diagana, T. , (2015). Perturbations of unbounded Fredholm linear operators in Banach spaces, in: Handbook on Operator Theory, 875-880, Springer Basel.

Diestel, J. , (1980). A survey of results related of the Dunford-Pettis property, Amer. Math.

Soc. of Conf. on integration, Topology and Geometric in Linear Spaces, 15-60.

Dominguez Benavides, T. , (1986). Some properties of the set and ball measures of noncompactness and applications, J. London Math. Soc., 34(2), 120-128.

Dunford, N. , \& Pettis, B. J. , (1940). Linear operators on summable functions, Trans. Amer. Math. Soc., 47, 323-392.

Dunford, N. , \& Schwartz, J. T. , (1971). Linear Operators, Part III, Wiley-Interscience, New York.

Edmum, D. E. , \& Evans, W. D. , (1987). Spectral Theory and Differential Operators, Oxford Science Publications.

Elleuch, A. , \& Jeribi, A. , (2016). On a characterization of the structured Wolf, Schechter and Browder essential pseudospectra, Indag. Math., 27, 212-224.

Elleuch, A. , \& Jeribi, A. , (2016). New description of the structured essential pseudospectra, Indag. Math., 27, 368-382.

Faierman, M. , Mennicken, R. , \& Muller, M. , (1995). A boundary eigenvalue problem for a system of partial differential operators occurring in magnetohydrodynamics, Math. Nachr., 173, 141-167.

Fakhfakh, F. , \& Mnif, M. , (2009). Browder and semi-Browder operators and perturbation function, Extr. Math., 24(3), 219-241.

Gethner, R. M. , \& Shapiro, J. H. , (1987). Universal vectors for operators on space of holomorphic functions, Proc. Am. Math. Soc., 100, 281-288.

Gohberg, I. C. , \& Krein, G. , (1960). Fundamental theorems on deficiency numbers, root numbers and indices of linear operators, Amer. Math. Soc. Transl., Ser. 2, 13, 185-264.

Gohberg, I. C. , \& Krein, M. G. , (1969). Introduction to the theory of linear non-self-adjoint operators in Hilbert space, Amer. Math. Soc., Providence.

Gohberg, I. , Markus, A. , ad Feldman, I. A. , (1967). Normally solvable operators and ideals associated with them, Proc. Amer. Math. Soc. Tran. Ser. 2(61), 63-84. 
Goldberg, S. , (1966). Unbounded Linear Operators, New York: McGraw-Hill.

Goldberg, S. , (1974). Perturbations of semi-Fredholm operators by operators converging to zero compactly, Proc. Amer. Math. Soc., 45, 93-98.

Gonzalez, M. , \& Onieva, M. O. , (1985). On Atkinson operators in locally convex spaces, Math. Z., 190, 505-517.

Grothendieck, B. , (1953). Sur les applications linéaires faiblement compactes d'espaces du type C(K), Canad. J. Math., 5, 129-173.

Gustafson, K. , \& Weidmann, J. , (1969). On the essential spectrum, J. Math. Anal. Appl., 25, 121-127.

Harrabi, A. , (1998). Pseudospectre d'une suite d'opérateurs bornés, RAIRO Modél. Math. Anal. Numér., 32, 671-680.

Hinrichsen, D. , \& Kelb, B. , (1993). Spectral value sets: a graphical tool for robustness analysis, Systems Control Lett., 21(2), 127-136.

Jeribi, A. , (1997). Quelques remarques sur les oprateurs de Fredholm et application l'quation de transport, C. R. Acad. Sci., Paris, Srie, I 325, 43-48.

Jeribi, A. , (1998). Quelques remarques sur le spectre de Weyl et applications, C. R. Acad.

Sci., Paris Sr. I Math., 327(5), 485-490.

Jeribi, A. , (1998). Développement de certaines propértés fines de la théorie spectrale et applications à des modèles monocinétiques et à des modèles de Reggeons, Thesis of Mathematics. University of Corsica, Frensh (16 Janvier 1998).

Jeribi, A. , (2000). Une nouvelle caractrisation du spectre essentiel et application, C. R. Acad. Sci. Paris, t. 331, Srie I, pp. 525-530.

Jeribi, A. , (2002). A characterization of the Schechter essential spectrum on Banach spaces and applications, J. Math. Anal. Appl., 271(2), 343-358.

Jeribi, A. , (2002). Some remarks on the Schechter essential spectrum and applications to transport equations, J. Math. Anal. Appl., 275(1), 222-237.

Jeribi, A. , (2002). A characterization of the essential spectrum and applications, Boll. dell. Unio. Mate. Ital., 8 B-5, 805-825.

Jeribi, A. , (2002). On the Schechter essential spectrum on Banach spaces and applications, Facta Univ. Ser. Math. Inform., 17, 35-55.

Jeribi, A. , (2003). Time asymptotic behavior for unbounded linear operator arising in growing cell populations, Nonl. Anal. Real Worl. Appli., 4, 667-688.

Jeribi, A. , (2004). Fredholm operators and essential spectra, Arch. Inequal. Appl., 2(2-3), 123-140.

Jeribi, A. , Krichen, B. , \& Zarai Dhahri, M. (2016). Essential spectra of some of matrix operators involving y-relatively bounded entries and an applications, Linear and Multilinear Algebra, 1654-1668.

Jeribi, A. , \& Latrach, K. , (1996). Quelques remarques sur le spectre essentiel et application l'quation de transport, C. R. Acad. Sci. Paris, t. 323, Srie I, 469-474.

Jeribi, A. , Latrach, K. , \& Megdiche, H. , (2005). Time asymptotic behavior of the solution to a Cauchy problem governed by a transport operator, J. Inte. Equa. Appl., Volume 17, Number 2, 121-139.

Jeribi, A. , Megdiche, H. , \& Moalla, N. , (2005). On a transport operator arising in growing cell populations II. Cauchy problem, Math. Meth. Appl. Scie., 28, 127-145.

Jeribi, A. , \& Mnif, M. , (2005). Fredholm operators, essential spectra and application to transport equations, Acta Appl. Math., 89(1-3), 155-176.

Jeribi, A. , \& Moalla, N. , (2006). Fredholm operators and Riesz theory for polynomially compact operators, Acta Appl. Math., 90(3), 227-245.

Jeribi, A. , \& Moalla, N. , (2009). A characterization of some subsets of Schechter's essential spectrum and application to singular transport equation, J. Math. Anal. Appl., 358, 434-444.

Jeribi, A. , Ould Ahmed Mahmoud, S. A. , \& Sfaxi, R. , (2007). Time asymptotic behavior for a one-velocity transport operator with Maxwell boundary condition, Acta Appl. Math., 3, 163-179.

Jeribi, A. , Moalla, N. , \& Walha, I. , (2009). Spectra of some block operator matrices and application to transport operators, J. Math. Anal. Appl., 351(1), 315-325. 
Jeribi, A. , Moalla, N. , \& Yengui, S. , (2014). S-essential spectra and application to an example of transport operators, Math. Methods Appl. Sci., 37(16), 2341-2353.

Jeribi, A. , Moalla, N. , \& Yengui, S. , (2017). Some results on perturbation theory of matrix operators, M-essential spectra of matrix operators and application to an example of transport operators, preprint.

Jeribi, A. , \& Walha, I. , (2011). Gustafson, Weidmann, Kato, Wolf, Schechter and Browder Essential Spectra of Some Matrix Operator and Application to Two-Group Transport Equation, Math. Nachr., 284(1), 67-86.

Kaashoek, M. A. , (1967). Ascent, descent, nullity and defect, a note on a paper by A. E. Taylor, Math. Ann., 172, 105-115.

Kaashoek, M. A. , \& Lay, D. C. , (1972). Ascent, descent and commuting perturbations, Trans. Amer. Math. Soc., 169, 35-47.

Karow, M. , (2006). Eigenvalue condition numbers and a formula of Burke, Lewis and Overton, Electron. J. Linear Algebra 15, 143-153.

Kato, T. , (1966). Perturbation Theory for Linear Operators, Die Grundlehren der mathematischen Wissenschaften, Band 132, Springer-Verlag New York, Inc., New York. Kato, T. , (1980). Perturbation Theory for Linear Operators, Second edition. Grundlehren der Mathematischen Wissenschaften, Band Springer-Verlag, Berlin Heidelberg, New York. Kulkarni, S. H. , \& Sukumar, D. , (2008). The condition spectrum, Acta Sci. Math. (Szeged), 74, 625-641.

Kumar, G. K. , \& Lui, S. H. , (2015). Pseudospectrum and condition spectrum, Operators and Matrices, 1, 121-145.

Langer, H. , Markus, A. , Matsaev, V. , \& Tretter, C. , (2003). Self-adjoint block operator matrices with non-separated diagonal entries and their Schur complements, J. Funct. Anal., 199(2), 427-451.

Latrach, K. , \& Dehici, A. , (2001). Fredholm, semi-Fredholm perturbations, and essential spectra, J. Math. Anal. Appl., 259(1), 277-301.

Latrach, K. , \& Jeribi, A. , (1996). On the essential spectrum of transport operators on L1spaces, J. Math. Phys., 37(12), 6486-6494.

Latrach, K. , \& Jeribi, A. , (1997). Sur une quation de transport intervenant en dynamique des populations, C. R. Acad. Sci. Paris, t. 325, Série I, pp. 1087-1090.

Latrach, K. , \& Jeribi, A. , (1998). Some results on Fredholm operators, essential spectra and application, J. Math. Anal. Appl., 225(2), 461-485.

Latrach, K. , Megdiche, H. , \& Jeribi, A. , (2005). Time asymptotic behavior of the solution to a Cauchy problem governed by a transport operator, J. Inte. Equa. Appl. 17(2), 121-140.

Lebow, A. , \& Schechter, M. , (1971). Semigroups of operators and measures of noncompactness, J. Func. Anal., 7, 1-26.

Leon-Saavedra, F. , \& Müller, V. , (2004). Rotations of hypercyclic operators, Inte. Equa. Oper. Theo., 50(3), 385-391.

Markus, A. S. , (1988). Introduction to the spectral theory of polynomial operator pencils, Amer. Math. Soc., Providence, RI. iv+250 pp. ISBN: 0-8218-4523-3.

Mennicken, R. , \& Motovilov, A. K. , (1999). Operator interpretation of resonances arising in spectral problems for $2 \times 2$ operator matrices, Math. Nachr., 201, 117-181.

M. M. Milovanović-Arandjelović , (2001). Measures of noncompactness on uniform spaces-the axiomatic approach, IMC Filomat 2001, Niš, 221-225.

Moalla, N. , (2012). A characterization of Schechter's essential spectrum by mean of measure of non-strict-singularity and application to matrix operator, Acta Math. Sci. Ser. B Engl. Ed., 32(6), 2329-2340.

Moalla, N. , Damak, M. , \& Jeribi, A. , (2006). Essential spectra of some matrix operators and application to two-group transport operators with general boundary conditions, J. Math. Anal. Appl., 323(2), 1071-1090.

Müller, V. , (2003). Spectral Theory of Linear Operator and Spectral Systems in Banach Algebras, Birkhäuser Verlag.

Müller, V. , (2007). Spectral theory of linear operators and spectral system in Banach algebras, Second edition, Operator Theory Advance and Application, vol. 139, Birkhäuser Verlag, Basel. 
Murray, F. J. , (1937). On complementary manifolds and projections in spaces Lp and Ip. Trans. Amer. Math. Soc., 41, 138-152.

Pelczynski, A. , (1965). On strictly singular and strictly cosingular operators. II. Strictly singular and strictly cosingular operators in $\mathrm{L}(\mu)$ - spaces, Bull. Acad. Polon. Sci. Sr. Sci. Math. Astronom. Phys., 13, 37-41.

Pelczynski, A. , (1965). On strictly singular and strictly cosingular operators. I. Strictly singular and strictly cosingular operators in $\mathrm{C}(\Omega)$-spaces, Bull. Acad. Polon. Sci. Sr. Sci. Math.

Astronom. Phys., 13 31-36.

Rako cevi'c, V. , (1981). On one subset of M. Schester's essential spectrum, Math. Vesnik., 5(18), 33(4), 389-391.

Rako cevi'c, V. R. , (1983). Measures of non-strict-singularity of operators, Math. Vesnik., 35, 79-82.

Rako cevi'c, V. , (1984). On the essential approximate point spectrum, 11, Math. Vesnik., 36, 89-97.

Rako cevi'c, V. , (1995). Semi-Fredholm operators with finite ascent or descent and perturbations, Amer. Math. Soci., 123(12), December.

Rako cevi'c, V. , (1998). Measures of noncompactness and some applications, Filomat, 12(2), 87-120.

Ransford, T. J. , (1984). Generalized spectra and analytic multivalued functions, J. London Math. Soc., 29, 306-322.

Reddy, S. C. , \& Trefethen, L. N. , (1990). Lax-stability of fully discrete spectral methods via stability regions and pseudoeigenvalues, Comput. Meth. Appl. Mech. and Engrg., 80(1), 147-164.

Riesz, F. , \& Nagy, B. S. , (1952). Leçons d'analyse fonctionnelle, Acadmie des Sciences de Hongrie, Akadmiai Kiadó, Budapest.

Schechter, M. , (1966). On the essential spectrum of an arbitrary operator, I. J. Math. Anal. Appl., 13, 205-215.

Schechter, M. , (1967). Basic theory of Fredholm operators, Anna. Scuola Norm. Sup., Pisa 21(3), 261-280.

Schechter, M. , (1968). Riesz operators and Fredholm perturbations, Bull. Amer. Math. Soc., 74, 1139-1144.

Schechter, M. , (1971). Spectra of Partial Differential Operators, North-Holland, Amsterdam. Schechter, M. , (1971). Principles of Functional Analysis, New Work: Academic Press.

Schechter, M. , (1972). Quantities related to strictly singular operators, Ind. Univ. Math. J., 21(11), 1061-1071.

Schmoeger, C. , (1997). The spectral mapping theorem for the essential approximate point spectrum, Colloq. Math. 74(2) 167-176.

Shapiro, J. , \& Snow, M. , (1974). The Fredholm spectrum of the sum and product of two operators, Trans. Amer. Math. Soc., 191, 387-393.

Shargorodsky, E. , (2009). On the definition of pseudospectra, Bull. London Math. Soc., 41, 524-534.

Shkalikov, A. A. , (1995). On the essential spectrum of some matrix operators, Math. Notes, 58(5-6), 1359-1362.

Shkalikov, A. A. , \& Tretter, C. , (1996). Spectral analysis for linear pencils $N-\lambda P$ of ordinary differential operators, Math. Nachr. 179, 275-305.

Taylor, A. E. , (1951). Spectral theory of closed distributive operators, Acta Math., 84, MR 12, 717, 189-224.

Taylor, A. E. , (1966). Theorems on ascent, descent, nullity, and defect of linear operators, Math. Annalen, 163, 18-49.

Trefethen, L. N. , (1990). Approximation theory and numerical linear algebra, Algorithms for Approximation II, Mason, J. C. , \& Cox, M. G. , eds., Chapman and Hall, London, 336-360. Trefethen, L. N. , (1992). Pseudospectra of matrices, numerical analysis. In: Pitman Research notes in Mathematics Series, Longman Science and Technology, Harlow, 260, 234-266.

Trefethen, L. N. , (1997). Pseudospectra of linear operators, SAIM rev., 39(3), 383-406. 
Trefethen, L. N. , \& Embree, M. , (2005). Spectra and Pseudospectra (The behavior of non normal matrices and operators), Princeton University Press.

Tretter, C. , (2008). Spectral Theory of Block Operator Matrices and Applications, Imperial College Press, London.

Varah, J. M. , (1967). The computation of bounds for the invariant subspaces of a general matrix operator, PhD thesis, Stanford University Pro. Quest LLC, Ann. Arbor.

Vladimirskii, Ju. I. , (1967). Strictly cosingular operators, Soviet. Math. Dokl. 8, 739-740.

West, T. T. , (1987). A Riesz-Schauder theorem for semi-Fredholm operators, Proc. Roy. Irish Acad. Sect. A, 87(2), 137-146.

Weyl, H. , (1909). Uber beschrankte quadratische Formen, deren differenz vollstelig ist., Rend. Circ. Mat. Palermo 27, 373-392.

Wilkinson, J. H. , (1986). Sensitivity of eigenvalues II, Util. Math., 30 243-286.

Wolf, F. , (1959). On the invariance of the essential spectrum under a change of boundary conditions of partial differential boundary operators, Indag. Math., 21, 142-147.

Wolff, M. P. H. , (2001). Discrete approximation of unbounded operators and approximation of their spectra, J. Approx. Theo., 113, 229-244.

Yood, B. , (1951). Properties of linear transformations preserved under addition of a completely continuous transformation, Duke Math. J., 18, 599-612.

Živković-Zlatanović, S. Č. , Djordjević, D. S. , \& Harte, R. E. , (2011). On left and right Browder operators, J. Korean Math. Soc., 48, 1053-1063.

Živković-Zlatanović, S. Č. , Djordjević, D. S. , \& Harte, R. E. , (2011). Left-right Browder and left-right Fredholm operators, Inte. Equa. Oper. Theo., 69, 347-363.

Živković-Zlatanović, S. Č. , Djordjević, D. S. , Harte, R. E. , \& Duggal, B. P. , (2014). On polynomially Riesz operators, Filomat, 28, 197-205. 